\title{
Number, colour and kinship in \\ New Zealand Sign Language
}

\author{
Rachel McKee
}

\section{NZSL and its lexicon}

The domains of number, colour and kinship in New Zealand Sign Language (NZSL) highlight diachronic variation and change in the lexicon. A brief outline of NZSL's history provides context for this variation.

Overlap in the lexicons of NZSL, Auslan and BSL evinces a close dialectal relationship between these varieties of the 'BANZSL' family (Johnston 2003; McKee and Kennedy 2000; Schembri et al. 2010; Woll 1987). But the relationship between them is not a straightforward divergence from a common mother language (BSL) through time and geographical separation. External intervention via the deaf education system, and ongoing contact between deaf nationals of these three nations have also had an effect. Although some 'early' NZSL signs are clearly of BSL origin, possibly brought by adult deaf immigrants from the UK, contact with BSL in the period following colonisation of New Zealand from the 1860s onwards was probably limited by the distance between northern and southern hemispheres. In the late 19th century, before a deaf school was established in the new colony, some New Zealand children were sent abroad to residential deaf schools in Australia, in which British and Irish sign languages were used, (Collins-Ahlgren, 1989; Fitzgerald 1999). It is known that at least one British-trained, hearing teacher of the deaf was employed as a tutor to a large family of deaf children in New Zealand during the 1870s, and used British signs with them (CollinsAhlgren, 1989). But to a large extent, it seems that the early generations of deaf people in New Zealand created their own signs in the context of oralist residential schools dating from 1880 onwards.

Convergence between NZSL and Auslan vocabulary increased sharply with the introduction of Australasian ${ }^{1}$ Signed English (ASE) into deaf education in both countries from the 1970s (1979 in New Zealand). The consensus among educators at the time was that the New Zealand sign lexicon was not extensive enough for use in education (Dugdale 2000). Vocabulary was 
drawn mainly from Auslan, which included some BSL-origin signs that were also in the NZSL lexicon. Sets of number, colour and kinship signs (among others) selected for the ASE system were adopted from Auslan used in the state of Victoria (Jeanes, Jeanes and Reynolds 1982), much of which had historical origins in BSL (Johnston \& Schembri, 2007).

At the time that ASE was introduced, NZSL lexicon in these domains was limited. Polysemous or generic manual signs were used to indicate semantic categories such as colour, month, relative, and city, with reference specified by the mouthing of an English word (Levitt 1985, McKee and McKee 2001, McKee and McKee 2007). The early lexicon of NZSL has similarities with systems described for other small-scale or emerging languages. A paradigm of context-dependent, polysemous signs is also described by Hoyer (2007) in the emergent variety of Albanian Sign Language, and Nyst (2007) reports 'simultaneous manual-mouthing' constructions using generic signs in Adamorobe Sign Language in semantic domains such as colour, shape, size or kin. Fox (2007) notes the progressive differentiation of colour terms through time, in the lexical system of Al-Sayyid Bedouin, a young sign language with few specific colour signs. Washabaugh (1986) observed that highly context-dependent lexicon is characteristic of a sign language that exists in conditions of limited diffusion and limited inter-generational depth - descriptors which fit the demographic conditions for NZSL in the century preceding the introduction of ASE in 1979.

The domains of number, colour and kinship were substantially relexified post-1979. Recent analysis of lexical variation shows that signs from the earlier paradigm are retained, particularly by older signers, and that some local innovations have also appeared (McKee, McKee and Major 2011). The NZSL number system today is similar to, but distinct in certain respects from the neighbouring language Auslan. Colour and kinship terms have also converged with Auslan in the last 30 years. The typology of number, colour and kinship domains described here for NZSL therefore reflects hybridity between an original paradigm that was not highly specified, and a modern lexicon that is more specified and morphologically elaborated.

\section{Number}

Expression of number concepts in NZSL exhibits considerable sociolinguistic variation, for the reasons outlined above. A dictionary of NZSL published in 1997 (Kennedy, Arnold, Dugdale, Fahey and Moskovitz 1997) records thirty forms representing the numerals zero to twenty. A previous dictionary 
(Levitt 1986) records a further seventeen signs for numbers between one to twenty that are not in the 1997 dictionary - practically an entire alternate set, including an 'air-writing' (tracing) form for numbers eleven to twenty. The strategy of tracing letters and numbers (above ten) is still used by some elderly signers rather than conventional fingerspelling or number systems (Collins-Ahlgren 1989; Forman 2003). A third scholarly description of NZSL (Collins-Ahlgren 1989) describes yet other number signs that appear in neither of the other two publications. The only numbers that are identically represented in these three lexicons are one, two, four and five, although all publications were based on reliable informant data collected within approximately one decade of each other. Air-writing (or tracing), and some earlier two-handed forms of numbers 6 to 10 found in the 1986 and 1989 publications that are now considered rare or archaic are not included in the 1997 dictionary. All entries in the 1997 dictionary were validated by ninety individuals from a representative cross-section of the deaf community (Kennedy, Arnold, Dugdale, Fahey and Moskovitz 1997); this suggests that at the time of that research, earlier expressions of number (those recorded in Levitt and Collins-Ahlgren's preceding work) were no longer regarded by the community as signs in common use.

\subsection{Cardinal numbers}

The NZSL cardinal number system reflects number concepts in the English language, including a base ten structure in the ordering of larger and smaller units (as also described for BSL by Skinner 2007:60). A 'zero' sign expresses negative number and quantity and is also used to express negative existence or to negate a predicate. As in most varieties of BSL and in Auslan, cardinal numbers are formed with extended digits, starting from the extended index finger as one, and index and middle finger for two. Number three has variant forms using three different combinations of adjacent extended fingers; the most frequent is the index, middle, ring finger combination.

Cardinal or counting numbers are made with the palm facing the signer, except for a variant of 10 which flicks the fingers palm outwards. For numbers $1-5$, the fingers point upright, while in numbers $6-9$ and $11-19$, the blade is down, fingers pointing sideways to the left (except earlier variants of 11 and 12 which have a different form).

Numbers six to ten are also produced with one hand, palm facing the signer, although an older variant of ten uses two 'five' hands. Numbers zero to ten are shown in figure 1. 


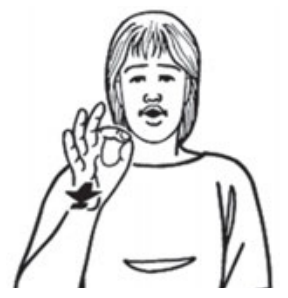

zero

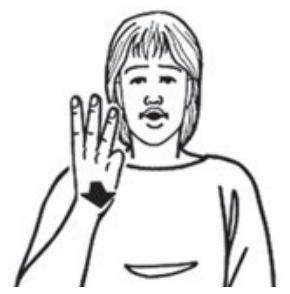

three

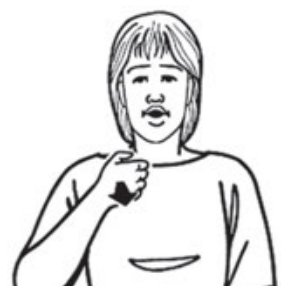

six (Aus)

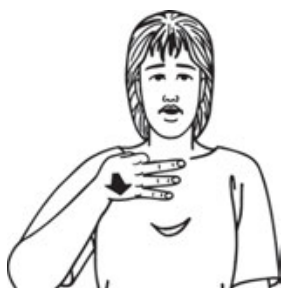

eight

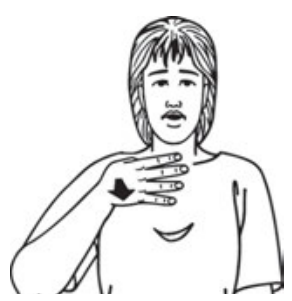

nine (early)

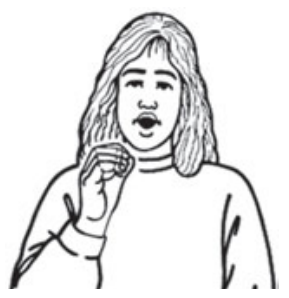

zero

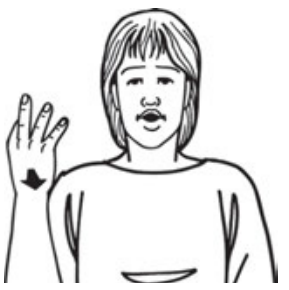

three

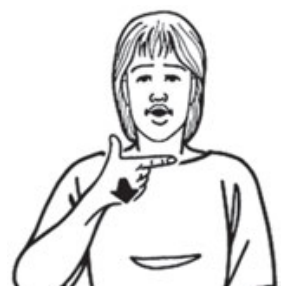

seven (Aus)

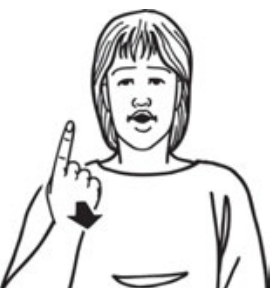

one

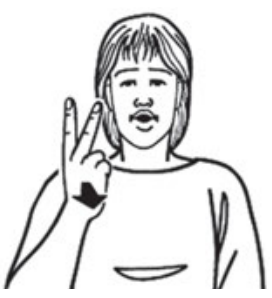

two

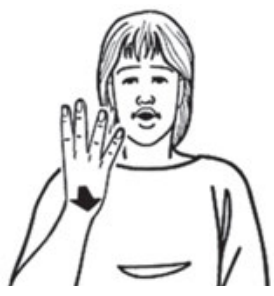

four

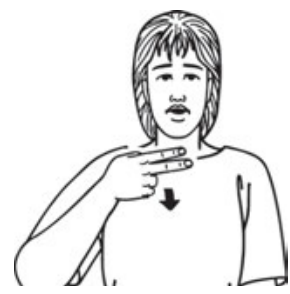

seven (early)

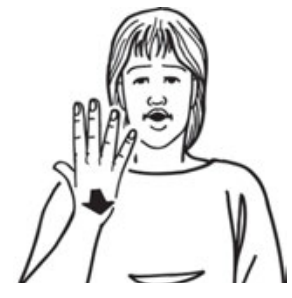

five

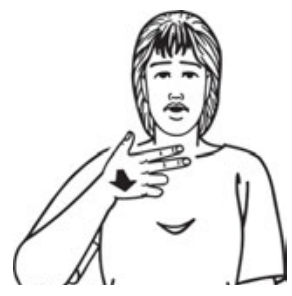

eight (Aus)/three

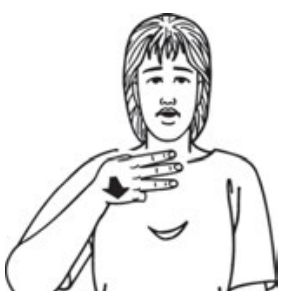

eight (early)

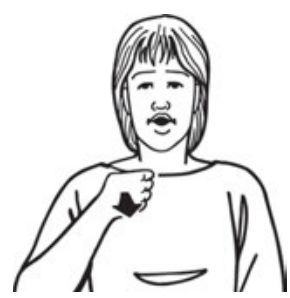

eight

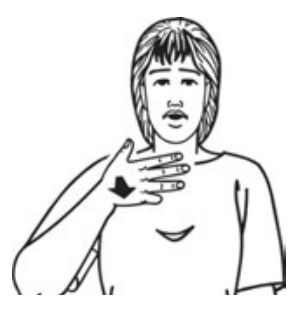

nine (Aus)
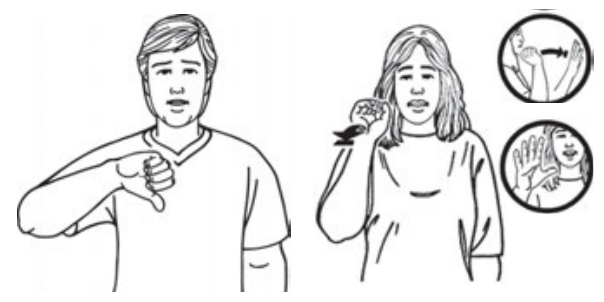

ten (Aus)

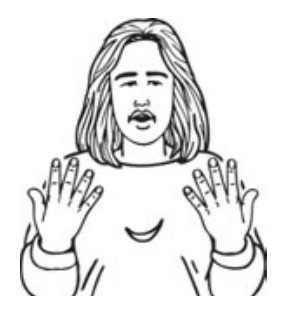

ten (early)

Figure 1. Cardinal numbers 0-10 (including variants) 


\subsection{Iconicity in cardinal numbers}

The number of extended fingers in signs for one to five, and the two-handed ten iconically represent number meaning. These signs are transparent to non-signers and overlap with counting gestures used by hearing people in New Zealand. Two-handed forms of six to ten (non-dominant five hand, plus extended digits on dominant hand), used more by signers over the age of 65, are also transparent in this way. However, representation is not entirely literal for numbers above five: for example, an optional 'five' non-dominant hand may be overtly expressed or dropped in some variants of numbers between six and ten (cf. Skinner 2007). The handshapes of modern forms of six (Auslan), seven (Auslan), a variant of nine ('thumb down'), and a variant of eleven (two fingers tracing vertical lines, see figure 3 ) have an element of iconicity possibly motivated by the shape of written numerals. Whether the handshapes of numbers six and seven are actually motivated by written numbers is unclear, since they also fit into a sequence of 'five plus-onedigit', and 'five-plus-two-digit' structure, followed by eight and nine in the same format.

As seen in figure 1, homophonous variants of three and eight exist; that is, the same three fingers used by some signers to mean 'three', may be used by other signers to mean 'eight', and vice-versa. Some signers maintain a distinction in meaning between three and eight through finger orientation - three points upwards, while eight points sideways (following the two-handed system for dominant hand digits in 6-9); some individuals are observed to use identical manual forms for three and eight, specified by mouthing.

\subsection{Cardinal numbers above 10}

NZSL has lexical signs for large number units above ten: hundred, thousand, and million (see figure 2). These can be combined with cardinal numbers to create complex numbers such as 400, 10,000, 100,000, expressed in the same word order as English. Multiples of a hundred are formed by moving a number handshape, (one to nine), under the chin, palm down. This form of number incorporation is borrowed from Auslan (Johnston and Schembri 2007). Number incorporation is not applied to the unit, thousand. 


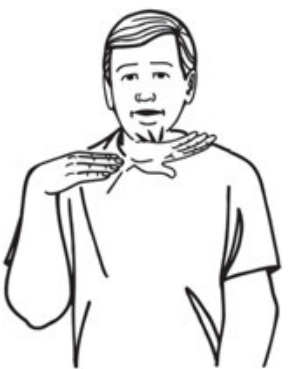

Hundred (Aus)

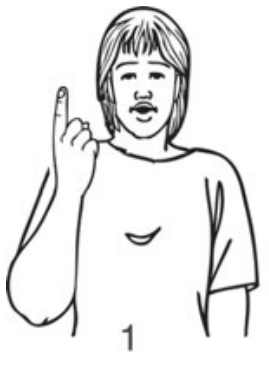

100 (Aus)

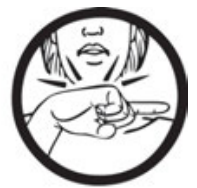

2

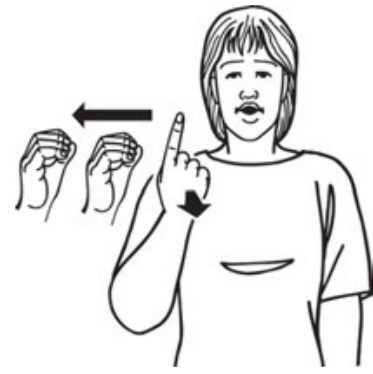

100 (early)

(+ number handshape)

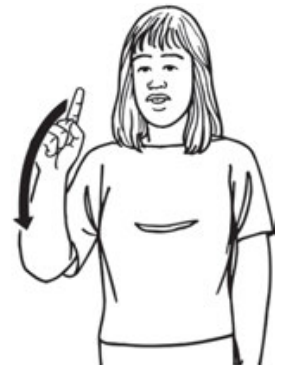

thousand

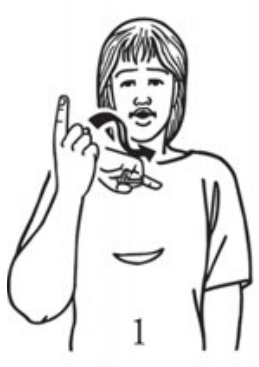

100,000
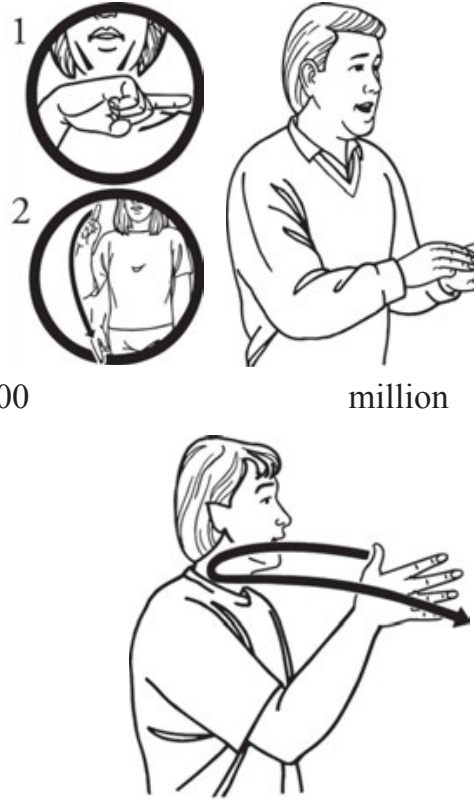

Five hundred/ Thousands/ Ages

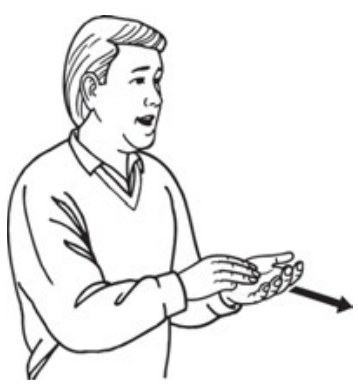

million

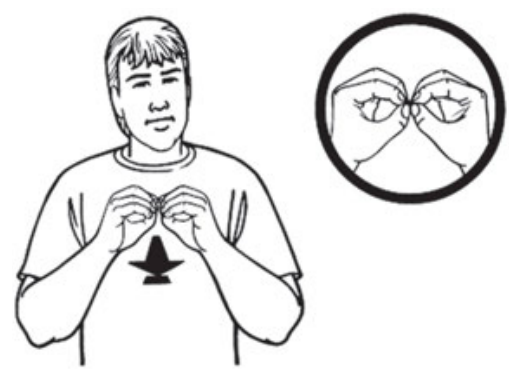

Billion

Figure 2. Large number units

Most of the signs shown in figure 2 were introduced with ASE, and correspond with Auslan signs. Earlier forms of hundred and thousand used a sequential compound of a cardinal number followed by reduplicated zero handshapes (eg, ONE-ZERO-ZERO), or a zero handshape moved laterally, or tracing zeros in the air. Some older signers do not express the zeros manually, but sign a cardinal number combined with mouthing to indicate a large number unit or nominal phrase, such as FIVE/ "thousand", or ONE/ "hundred yards"; in this system, meaning is retrieved from mouthing and context. Finally, one unique early sign expresses the sense 'thousands' or an 
unspecified huge number, as well as the temporal meaning of many years ('ages'). An almost identical form is used exclusively by older signers to mean 'five hundred' specifically. (Both are illustrated in Figure 2).

\subsection{Strategies for constructing cardinal numbers}

\subsubsection{Simple multiples of ten}

Multiples of ten comprise a sequential compound of a number plus zero; e.g., twenty is expressed as TWO-ZERO, thirty as THREE-ZERO, etc, up to ninety (see examples in figure 3). Both closed and open-hand ' 0 ' handshapes are used; the open-hand zero (ASL ' $F$ ') is an earlier form. The structure of ten multiples differs from that found in Auslan, in which a number handshape is combined with a change in orientation from palm towards the signer to 'palm-away' (outward rotating) movement. Older signers might alternatively trace two digit numbers in the air with the index finger.

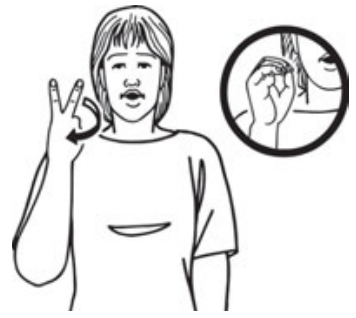

20

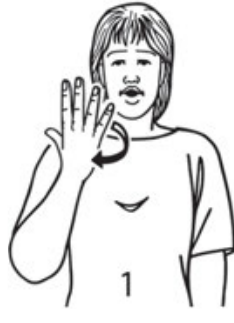

50

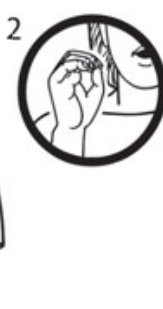

(1)

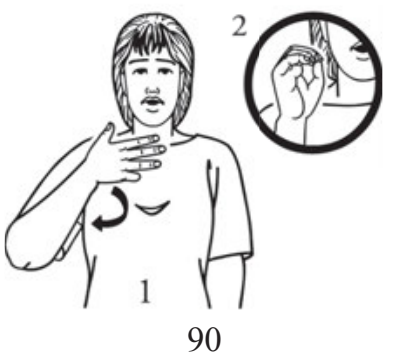

90

Figure 3. Multiples of ten

\subsubsection{Complex numbers above ten}

Single digit numbers between one and nine combine with a vertical shaking movement to express numbers 11-19 as used in Auslan (see examples in figure 4). NZSL therefore has a mixed paradigm that has adopted an Auslan system for expressing '-teen' numbers (including 11 and 12), but retained a sequential NUMBER ZERO structure for multiples of ten rather than the movement seen in Auslan (Johnston and Schembri 2007). 


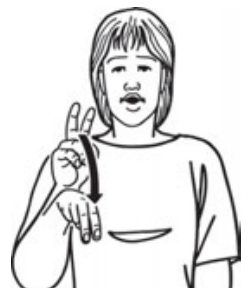

eleven (early)

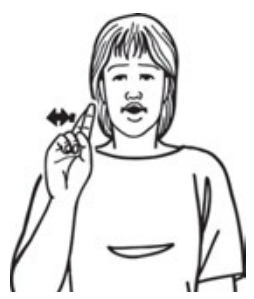

twelve

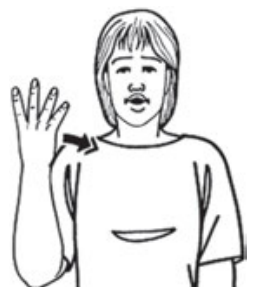

fourteen (Aus)

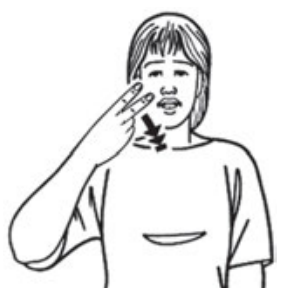

seventeen

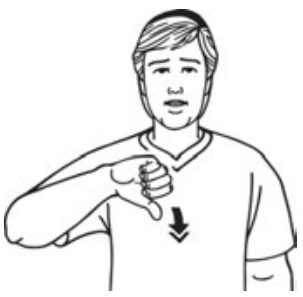

nineteen

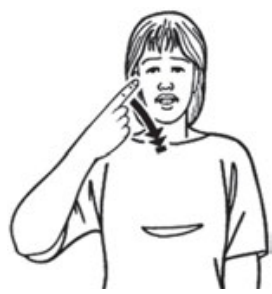

eleven (Aus)

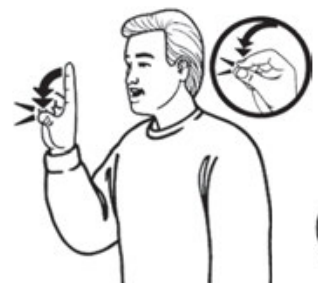

eleven (Aus)

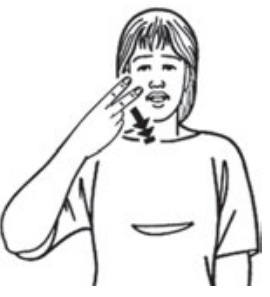

twelve (Aus)
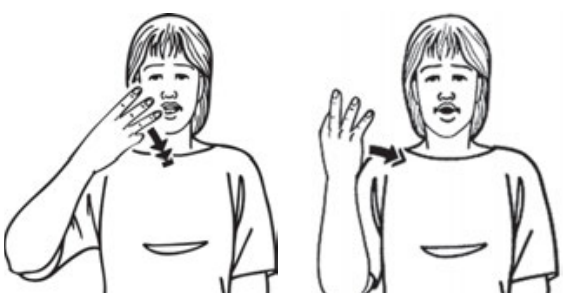

thirteen

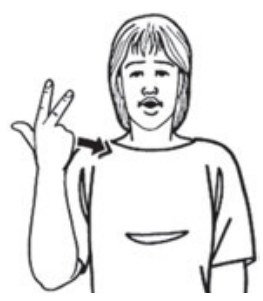

thirteen

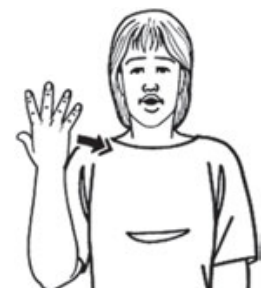

fifteen (Aus)

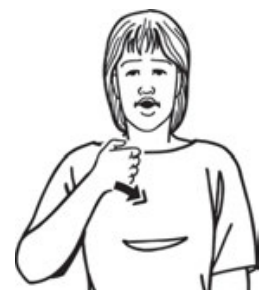

sixteen (Aus)

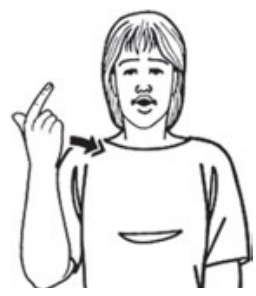

seventeen (Aus)

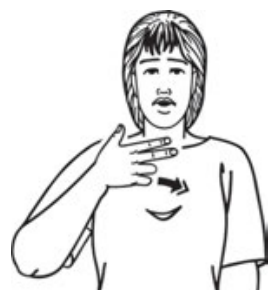

eighteen (Aus)

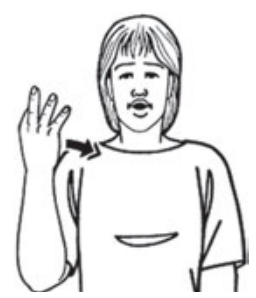

eighteen

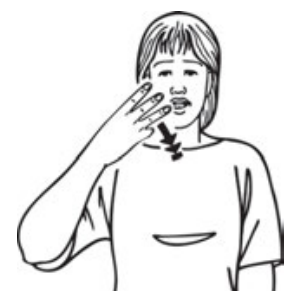

eighteen
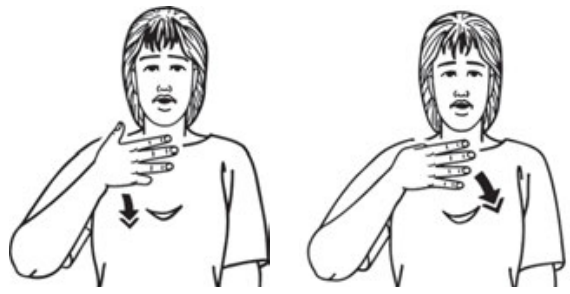

nineteen

Figure 4. eleven - nineteen 
Two-digit numbers $(21,22$, etc.,) use a compound sequence, moving in an outward direction from centre to dominant side.

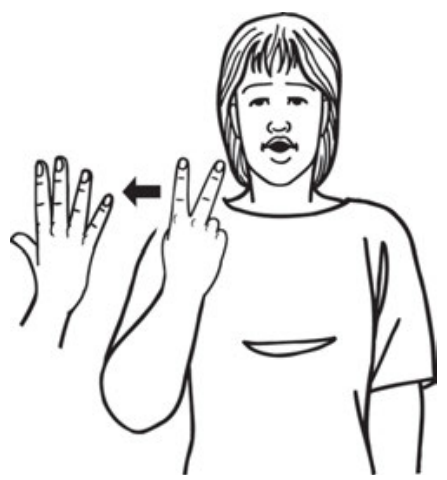

Figure 5. Two digit number (twenty five)

\subsubsection{Fractions}

Several strategies are used in NZSL to express fractional numbers and quantities. Lexical signs for half and quarter are the most longstanding terms for fractions; these are shown in figure 6. These signs are also used with reference to clock time (half past, quarter past, quarter to); the sign 'quarter' is apparently motivated by reference to a horizontal line between three and nine on a clockface (a quarter before or after the hour). 'Half' has variant forms: (a) a flat blade drawn across the open palm, (b) a horizontal index finger drawn across an upright index finger at the middle knuckle (which has a secondary translation of 'quarter' in a dictionary entry), and (c) a flat blade drawn across the signer's midriff (an older form not recorded in NZSL dictionaries). An early sign for 'three-quarters' corresponds with the (b) form of 'half' - in which the index finger is drawn across the vertical index below the middle knuckle.

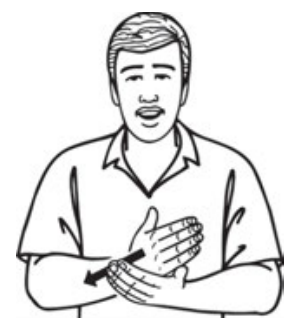

Half (a)/'half-past'

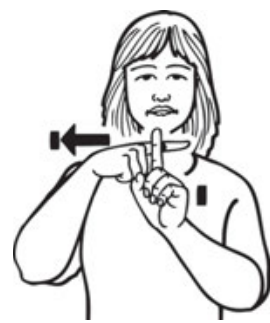

Half (b)

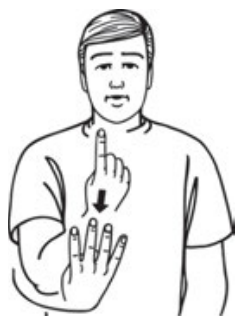

one-quarter

Figure 6. Fractions 
Since signing has been used in the education system from the 1980s, signs motivated by mathematical orthography have been developed in the school system, and disseminated into community use. Columnar (a sequence of two numbers formed in a vertical arrangement) and decimal (number-POINTnumber) forms of fractions have emerged in this lexicon, (see figure 6). Established signs for percent are motivated by the symbol, as seen in figure 7 .

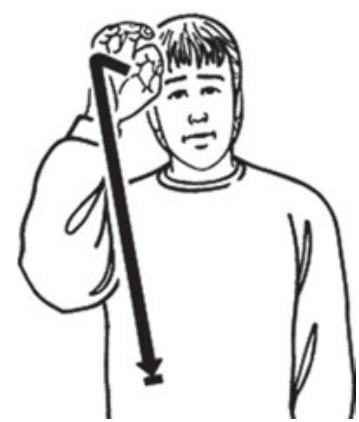

Percent 1

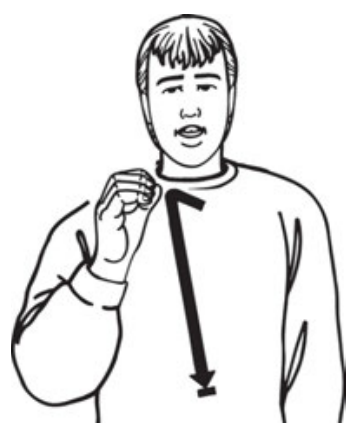

Percent 2

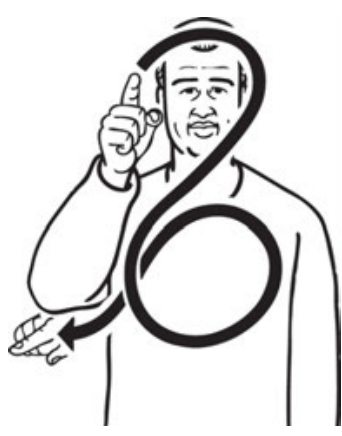

Percent (early)

Figure 7. Percent - variants

\subsubsection{Ordinal numbers}

Ordinal numbers 1st - 10th are formed by a movement inflection of a cardinal number: a single inward-facing twist or rotation of the wrist (see figure 8). Numbers above 10 are not inflected in this way, and the inflection is variably applied to 10 (also depending on the variant of ten used: the 'flicking' variant is more awkward to combine with a twisting movement than the 'two-handed-five' form). Ordinal meaning for numbers above ten (e.g., 'He came $12^{\text {thr }}$ ) is understood by context and sometimes mouthing.

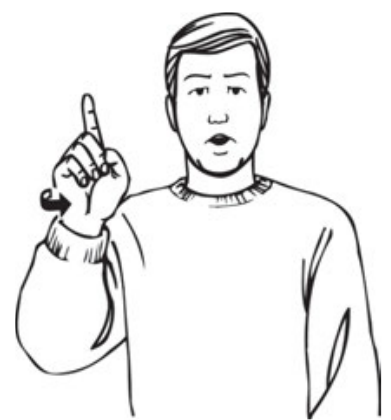

First

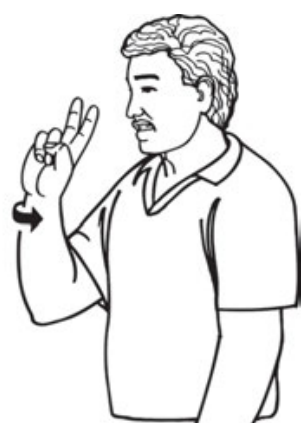

Second

Figure 8. Ordinal number examples 
For definite anaphoric reference to a member of an ordered set, ordinal meaning can be expressed by touching, or lightly grasping, the horizontal fingertip of non-dominant hand list buoys to express 'the first one', 'the second one', etc, (up to fifth), of an identified set. (See figure 9).

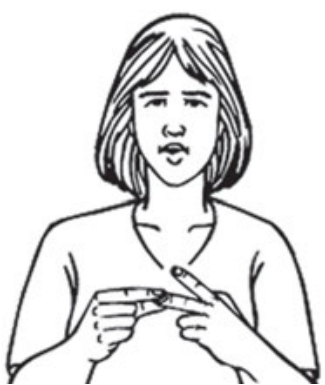

(the) second(one) secondly

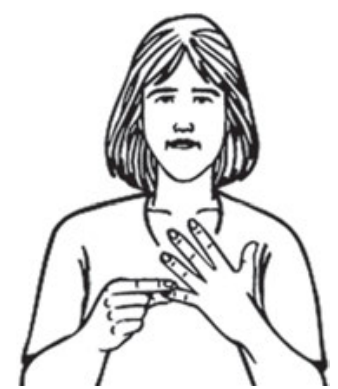

(the) fifth (one) fifthly

Figure 9. Ordinal list buoys

\subsection{Numeral Incorporation}

Cardinal numbers can be combined with movement and location inflections in semantic categories of personal pronouns, age, time, and money.

\subsubsection{Personal pronouns}

Number of persons between two and five can be specified in personal pronouns; these forms comprise the relevant number handshape moved in an alternating or circling pattern to indicate a grouping of persons. Inclusive or exclusive (of the signer) reference is indicated by proximity of the sign to the signer's body, as shown in figure 10 .

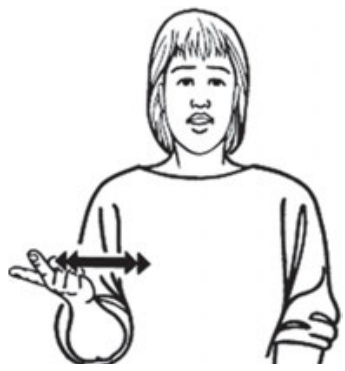

two of them

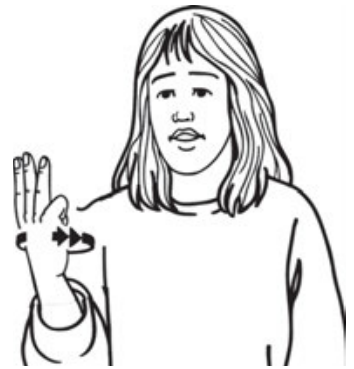

three of them/us

Figure 10. Numbered pronouns 
A classifier handshape (typically an upright index finger representing humans, horizontal index for animals) representing a body moving or a spatial arrangement can incorporate number up to five, by extending extra fingers.

\subsubsection{Age numbers}

Age numbers (for whole years) are made with the index finger of the number handshape touching the tip of the nose and moving outwards from the face (see figure 11). This originates in a compound of the sign AGE (located on the nose) with a number sign, and translates as 'x-years-old'. This number incorporation is most frequently seen with numbers up to ten (excluding the thumb-down 'nine' variant, which is awkward to articulate at a nose location), but is also possible with two digit numbers. The age of infants stated in number of weeks or months does not use this system; these are stated in a lexical phrase such as AGE SIX MONTH, or ELEVEN WEEK AGE.

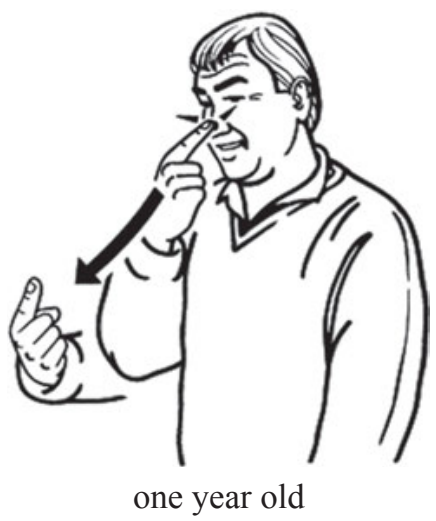

Figure 11. Years of age

\subsubsection{Clock time}

'O'clock' (on the hour) times are formed with a small swivelling movement of a number handshape in neutral space, made by rotating the wrist back and forth quickly (see figure 12). There are separate signs for fractional clock time - expressing 'quarter past', 'half past', 'quarter to', and 'minutes past' the hour; when these signs are used in a phrase (e.g., HALF SIX, QUARTER-TO FOUR, TEN PAST TWO ), the hour number in the phrase does not take the swivelling 'o'clock' movement. 


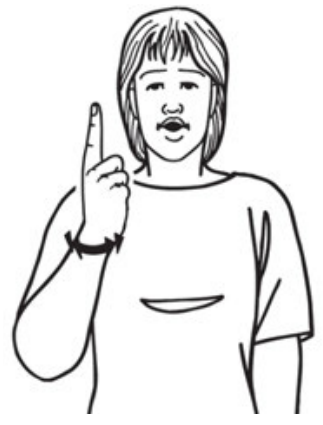

One o'clock

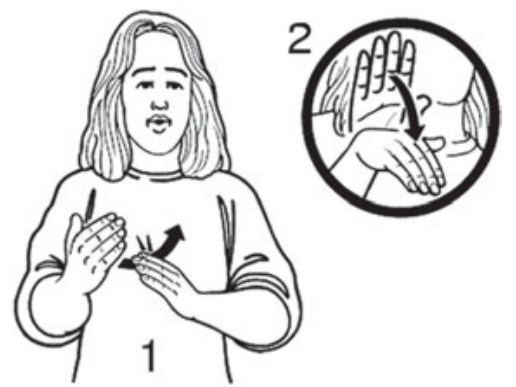

quarter past

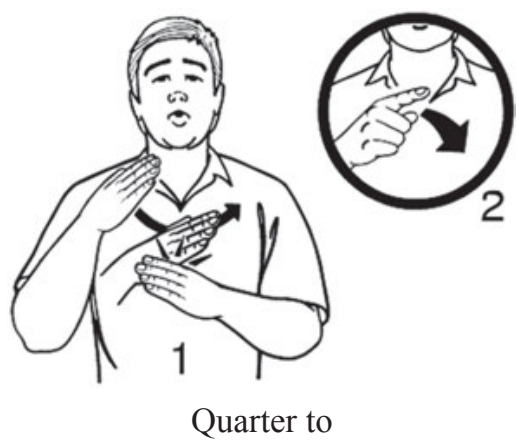

Figure 12. Clock time expressions

The phrasing of analogue clock time expressions are influenced by NZ spoken English, as seen in the use of signs meaning 'half past', 'quarter to', 'five to', 'ten past' etc. Digital time expressions (such as 'one forty five', or 'six fifteen') can also be signed in corresponding form - with a 'point' between the hour and minute numbers, reflecting a digital clock display; for example, ONE POINT FORTY. The digital format is increasingly common among younger signers, parallel with the same trend in spoken NZ English.

One variant of 'hour' (an extended upright index, palm back, moved in a clockwise circle in front of the signer's face) can incorporate a number handshape up to five. Signs for 'minute' and 'second' do not regularly incorporate number in NZSL.

\subsubsection{Calendar time}

Number handshapes can be combined with certain units of calendar time, including signs for WEEK (see figures 13a and b), YEAR, a sign that means future-time-period (week, month or year) (see figure 13c), past-WEEK/ 
YEAR, WEEKLY (see figure 13d) and YEARLY (see figure 13e). These can incorporate the handshape of numbers one to four, and rarely, five. Numbers between six to nine are occasionally combined with signs WEEK or YEAR, but are not generally combined with the movement morpheme signalling future or past time reference. Number incorporation is not used with signs for DAY or MONTH. Recurring time intervals (e.g., every two weeks, every three years) can incorporate a number between one to five into the movement of the sign week or year. A twisting movement inflection expresses the concept of 'once' and 'twice' (see figure $13 \mathrm{f}$ ).

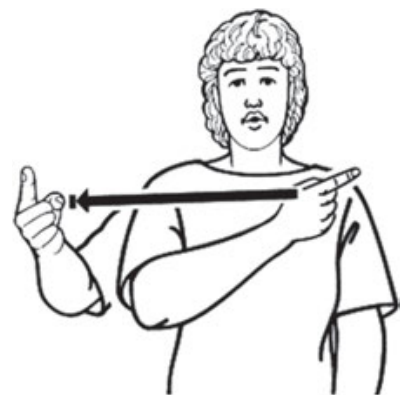

(a) One Week
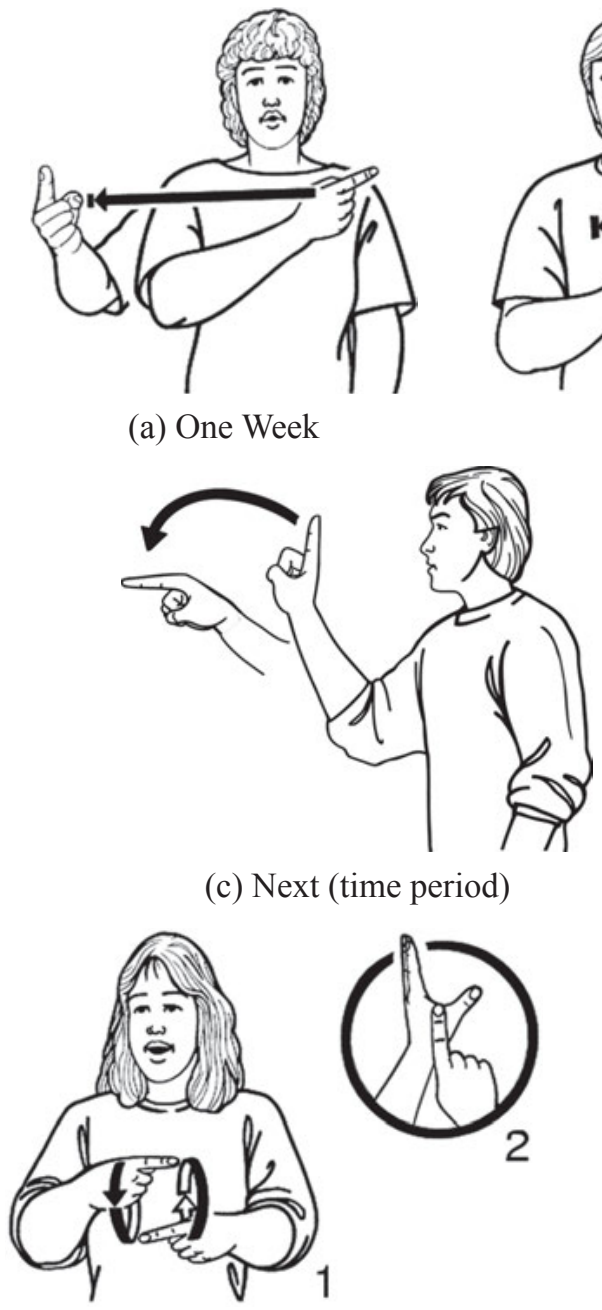

(e) Yearly

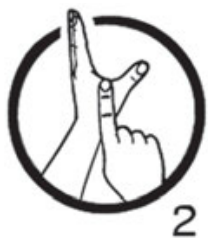

(c) Next (time period)

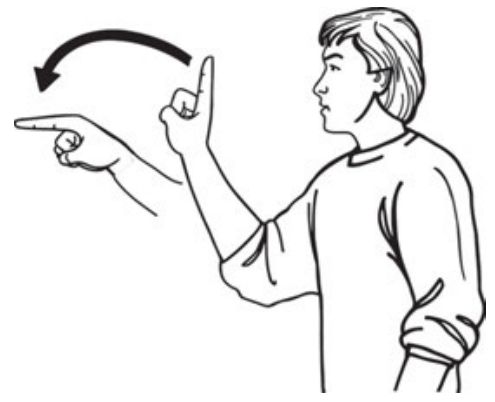

(b) Two weeks

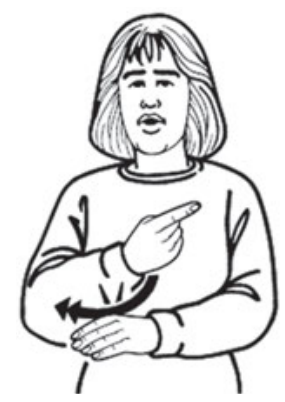

(d) Weekly

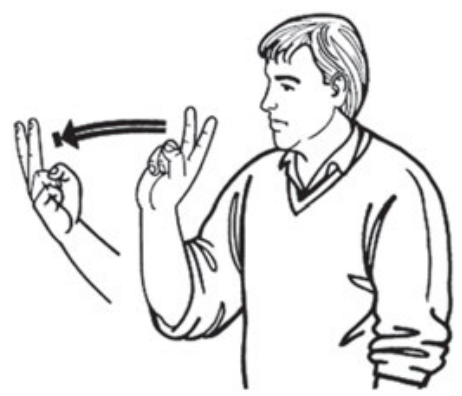

(f) Twice

Figure 13. Number incorporation in time units 


\subsubsection{Money numbers}

The expression of money numbers varies. In the traditional (still widely used) paradigm, money expressions use lexical phrases, such TEN DOLLAR, or the unit term (e.g., 'dollar') might be signalled in the mouthed phrase accompanying a number sign. Dollar and cents amounts can either be signed in full, such as FIVE DOLLAR SEVENTY C(ent), or FIVE DOLLAR SEVENTY, or, in decimal point form, as FIVE POINT SEVENTY.

Among younger signers, two number incorporating systems for whole dollar amounts are becoming established. These comprise (i) a movement modification of a number handshape (below ten), in which the extended fingers of a number handshape (between one and five) are flexed (see figure 14a), and (ii) a location modification in which a number handshape (below ten) contacts and moves out from the chin (see figure 14b). The first is apparently a local innovation, and the second appears to be a borrowing adapted from either contemporary BSL, which utilises this system for quantifying British pounds, or an earlier (pre-decimal) usage of this form in Auslan. ${ }^{2}$
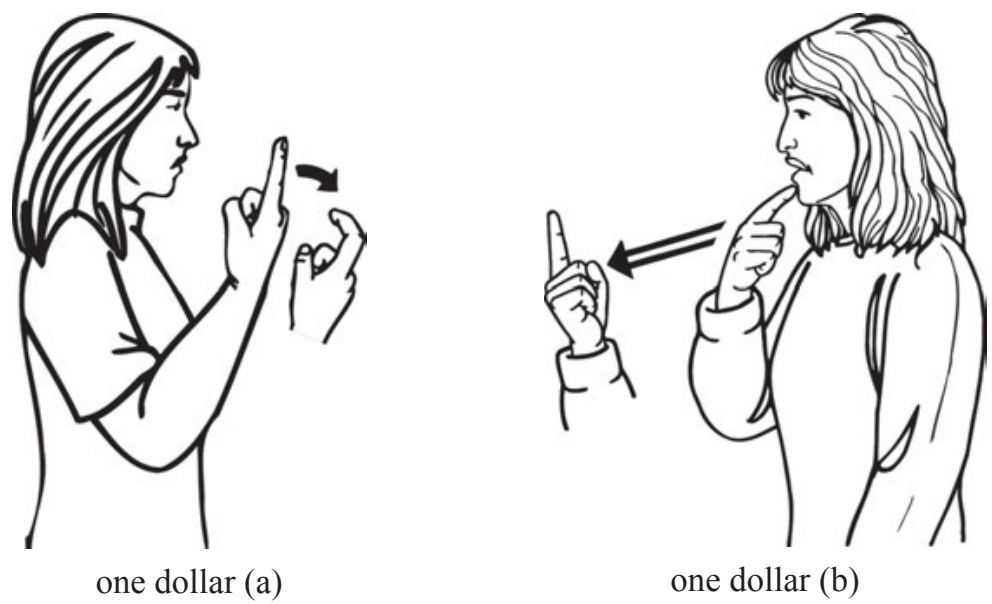

Figure 14. Number incorporation in money units

\subsection{Quantification}

\subsubsection{Nominal plurals}

A few nouns can be marked for pluralisation by individually articulated repetitions of the sign at distinct locations in the signing space. The conventionalised plural 'children' is one of few examples of this process in 
NZSL (figure 15). Reduplication for pluralisation is applied to few conventional nouns, and apparently only those articulated in neutral signing space, usually without body contact (e.g., PERSON - figure 15b, or PROBLEM). The most common strategy for quantification is use of lexical modifiers such as SOME, MANY, FEW, DIFFERENT, or relying on context to imply plural reference.

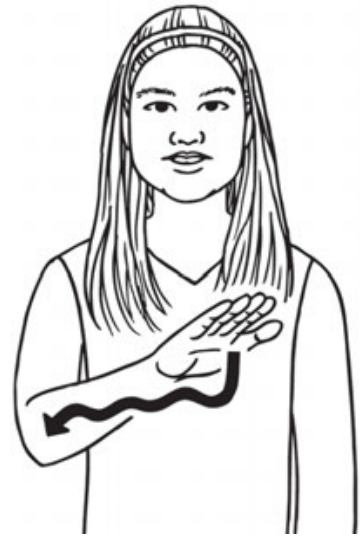

children

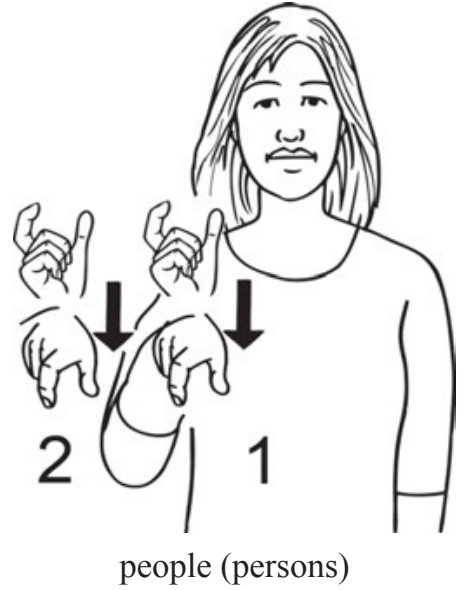

people (persons)

Figure 15. Lexicalised plurals

Productive (classifier) signs representing entities or shapes can be reduplicated in neutral space or on the body to express plurality, or placed more deliberately to convey specific number - e.g., a pattern of spots on a garment, a cluster of high-rise buildings (as illustrated in figure 16).

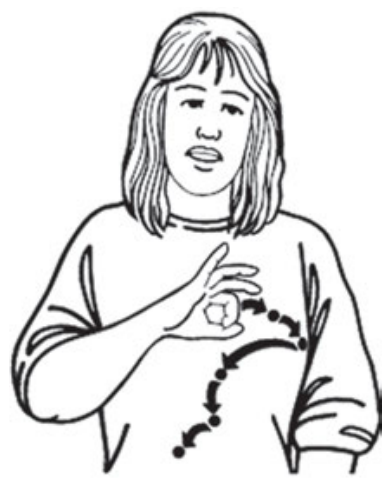

spots (multiple)

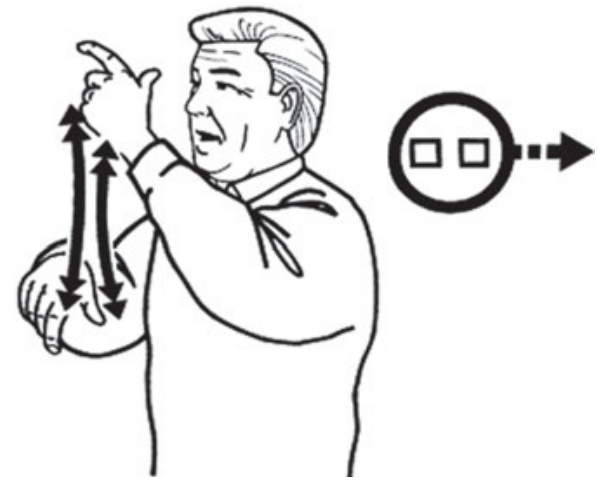

buildings

Figure 16. Reduplicative pluralisation in productive signs 
Some productive forms have become lexicalised as signs with internal pluralisation - e.g., 'exhibition' (multiple items on a wall), and 'queue' (multiple people); (see figure 17).

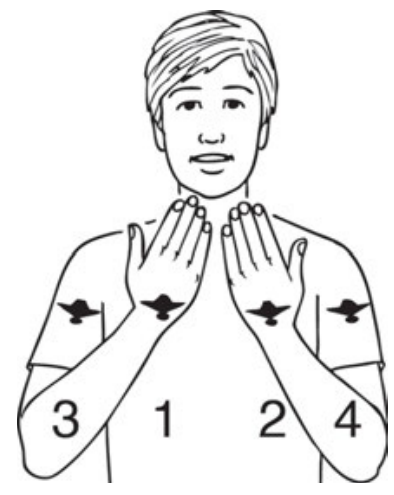

Exhibition

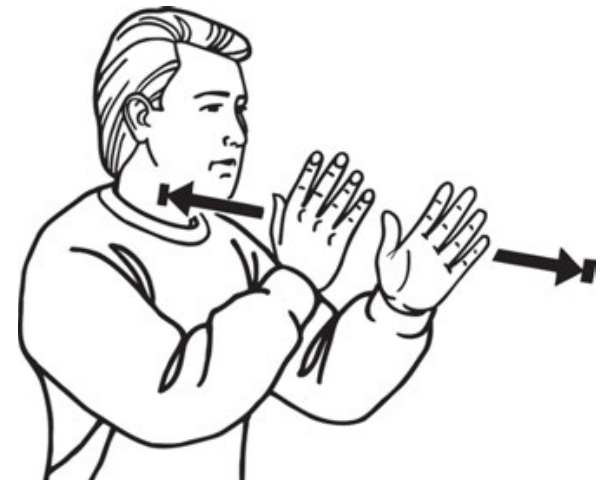

Queue

Figure 17. Lexicalised internal reduplication

The only identified suppletive plural form is person/people, (figure 18), for which neither of the plural forms is formally related to the singular form.

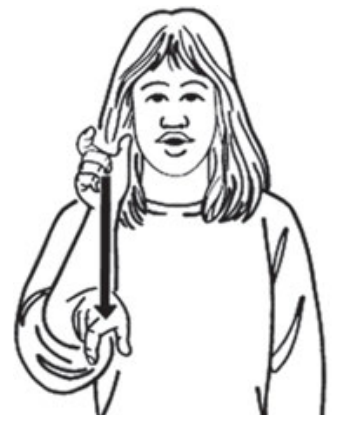

Person

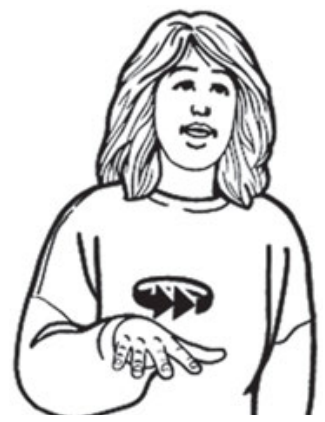

People 1 (early)

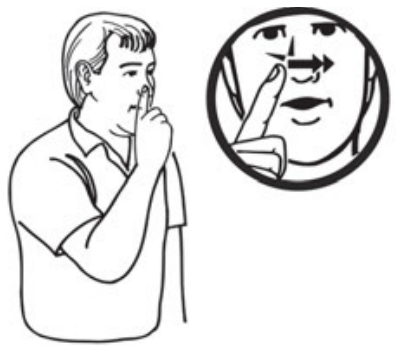

People 2 (Aus)

Figure 18. Suppletive plural 


\subsubsection{Inventory of quantifiers}

As mentioned above, lexical quantifiers can be used in nominal phrases. These are shown in figure 19.

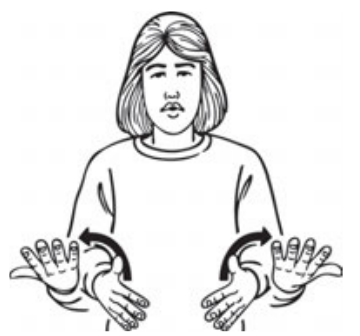

any/anything

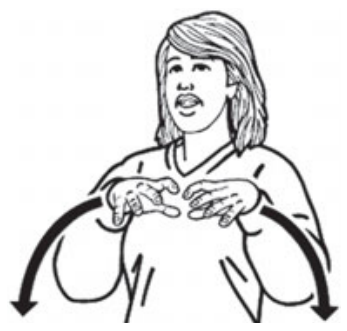

much
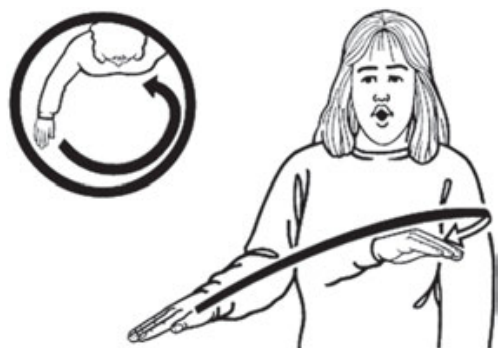

all

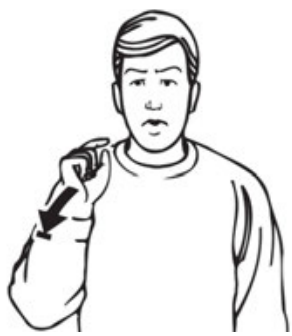

few/little

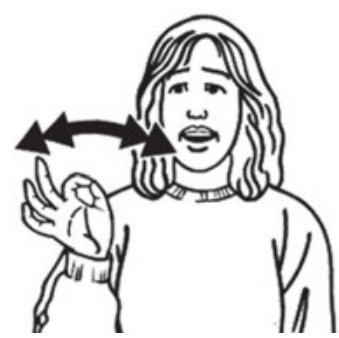

nothing/not-have

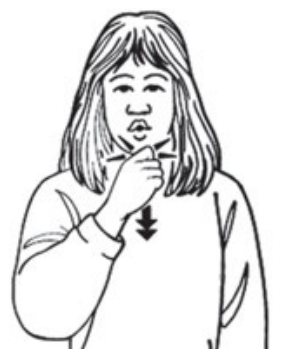

few/rare

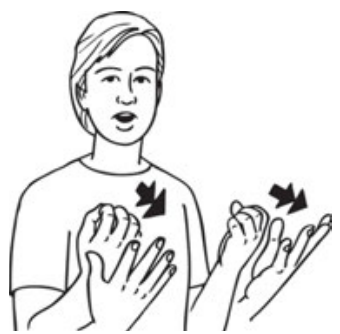

many/most

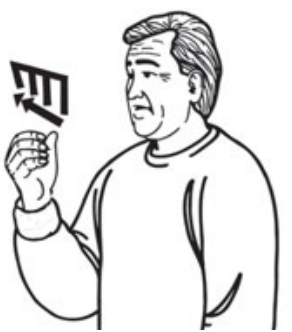

some

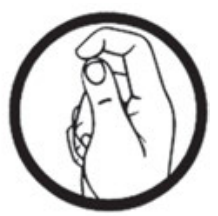

Figure 19. Lexical quantifiers

Negative quantifiers can be located in the signing space to indicate negative quantity or existence in relation to a discourse referent. Signs that are regularly translated as 'empty (place)' and 'no-one' are conventionalised examples of spatial modification; (see figure 20). 


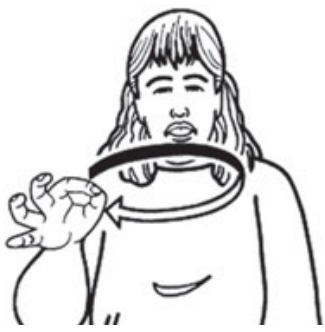

Empty, unoccupied (place)

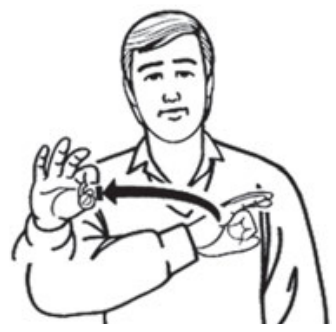

No-one

Figure 20. Negative quantifiers, spatially modified.

Quantifiers - some, few, and many - can be shifted in the signing space to index discourse referents in the signing space.

\section{Colour}

All available evidence indicates that the early (pre-ASE) paradigm for colour signs in NZSL comprised two manual signs: black (corresponding with the BSL and Auslan sign), and a generic sign that indicated any colour, specified by mouthing an English colour name illustrated in figure 21. In the first dictionary of NZSL (Levitt 1985), a signer of the older generation (in his mid-seventies at the time) is pictured modelling the generic colour sign in entries glossed as white, blue, yellow, red, green, brown, and interestingly, also black (suggesting that he and perhaps his peers actually used a single colour term). In this paradigm, colour reference requires knowledge of spoken English colour words, although mouthed articulations are not necessarily identical with those of hearing speakers. ${ }^{3}$ The generic sign also expresses the abstract category, colour. It is listed in the 1997 NZSL dictionary (Kennedy et al, 1997:196) with the translation, 'colour, shade', noting that the lip pattern is either "colour, or the name of a colour".

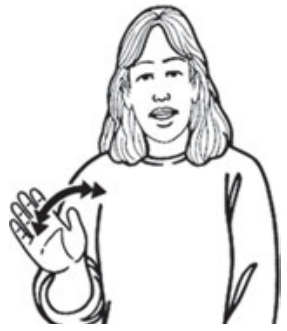

Colour(s) - generic

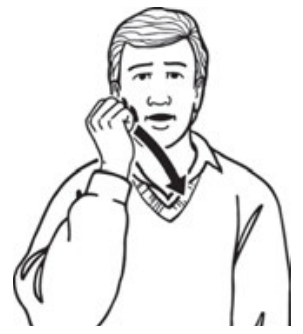

Black

Figure 21. Early colour signs 
As with numbers, the adoption of ASE (Auslan) vocabulary in 1979 brought an elaborated set of colour signs borrowed from Auslan, and a decline in the use of the generic colour sign. A current dictionary of NZSL (McKee et al, 2011) records 15 different colour signs, including some with two variants, as follows: black, blue, brown, green 1 and 2, grey, pink, purple, red 1 and 2, silver, white, yellow, colour, grey, orange 1 and 2. To the best of our knowledge, all of these except colour, black and one variant of green were introduced via ASE. These are illustrated in figure 22 (excluding close phonological variants of red and orange).

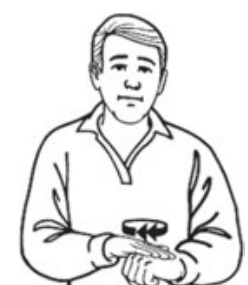

blue

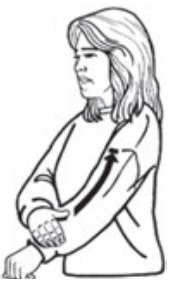

green

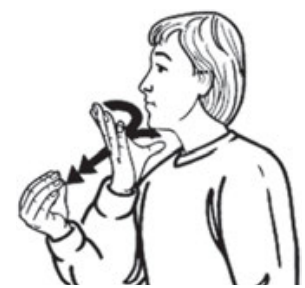

brown

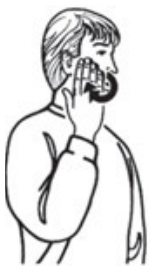

grey

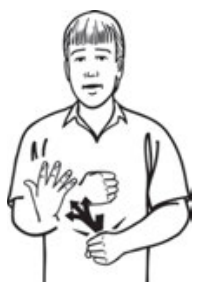

gold

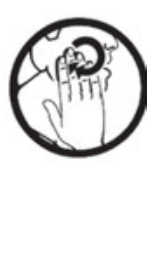

\section{)}

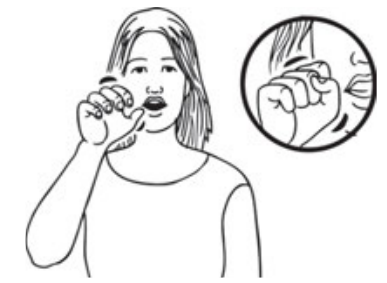

orange

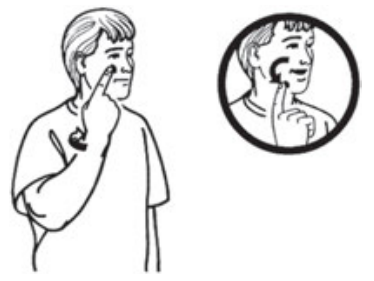

pink

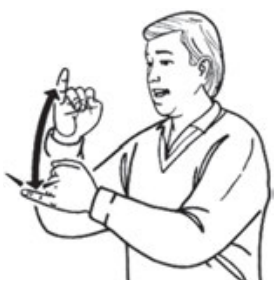

silver

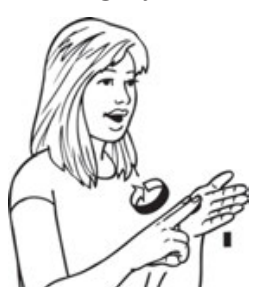

purple
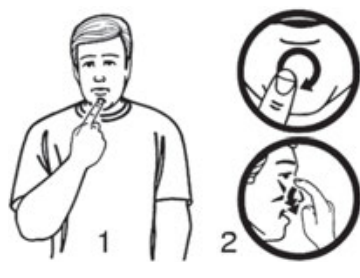

red
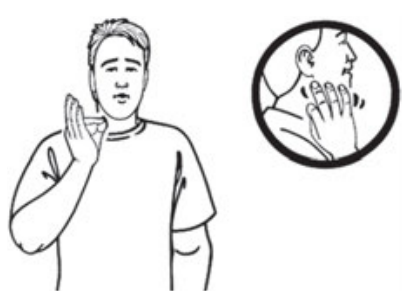

white

Figure 22. Modern colour signs (borrowed from Auslan) 
Colour signs disseminated via ASE were readily adopted not only in the school system, but also by the adult language community, as they supplemented an under-specified domain of the native lexicon. A study of sociolinguistic variation in NZSL confirms that use of the generic colour sign persists, but is confined largely to older signers; it was produced by most signers over 65 years old in response to six different colours shown in an elicitation task (McKee and McKee 2011). Among younger signers the meaning of the generic sign is restricted to the category term, 'colour', and is also used as a modifier following another colour sign to indicate an indeterminate or approximate shade, such as 'brownish'.

Even in the modern paradigm, the number of colour signs is small compared to English colour vocabulary; there are no established terms for intermediate colours such as turquoise, mauve, khaki, beige, maroon, nor for naming shades within a colour range that are distinguished in English, such as sky blue, royal blue, French blue, navy blue.

In Berlin and Kay's (1969) hierarchical typology of language and cultural complexity according to the extent of colour terms, a two-way colour distinction would place early NZSL as a Stage 1 language. Applying Berlin and Kay's typology to colour systems in signed languages can be problematic; firstly, many colour signs derive from reference to physical objects for colour identity (e.g., lips/red, teeth/white), a form of secondary reference which is excluded from their criteria for forming basic colour terms, and secondly, the conceptual and formational characteristics of colour terms in signed languages may be strongly shaped by contact with spoken language(s), (cf. Nyst 2007, and Hollman and Sutrop 2011). Contact notwithstanding, Stokoe (1987:10) points out that it cannot be assumed that a deaf community living within a society that has a more highly specified lexicon will necessarily develop a vocabulary and set of cultural referents that parallel the surrounding spoken language, since this may be inaccessible to deaf people. The limited set of early NZSL vocabulary in three core domains illustrates this point. Expression of colour concepts in NZSL was restricted for approximately a century in terms of native manual lexicon, but has interacted productively with the semiotic systems of other languages: initially, through mouthing of English words, and later, via adoption of a larger repertoire of Auslan signs, which parallel the main colour names in English. 


\subsection{Colour sign combinations and modifications}

To express intermediate or mixed shades, colour signs can be used in sequential combinations - such as BLUE GREEN, RED BROWN. Intensity of hue can be expressed by lexical modifiers DARK, BRIGHT and LIGHT, preceding or following a colour sign. As mentioned above, the generic colour sign may be used as a post-modifier to indicate an approximate shade ('-ish').

Colour signs are also modified for intensity (of saturation and brightness) by simultaneous non-manual signals. Degrees of darkness or lightness can both be signalled by eye-narrowing, more pronounced and with more brow frown for darkness. Eye-widening is associated with brightness. As colour signs are typically accompanied by a mouthed word (like most nouns and adjectives in NZSL), mouth modifiers are less consistently observed. Non-manual modifers are shown in figure 23. Movement of a colour sign may be tensed and slowed to express intensity of hue (e.g., 'very red'). Observation suggests that these non-manual modifications are not consistently applied by all signers; as with other morphological features, their use seems to vary by individual factors of fluency, nativeness, and age-group.

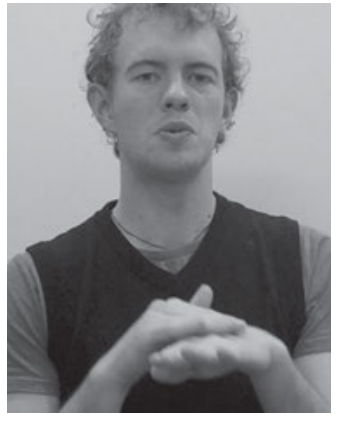

Blue unmodified

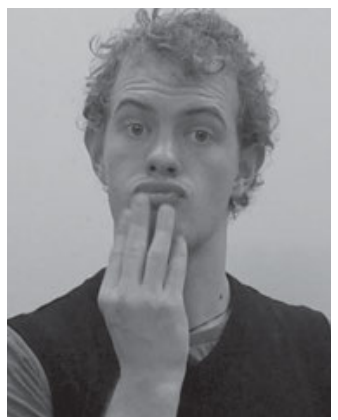

Brown unmodified

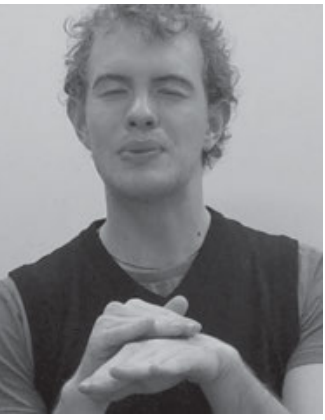

Blue - light

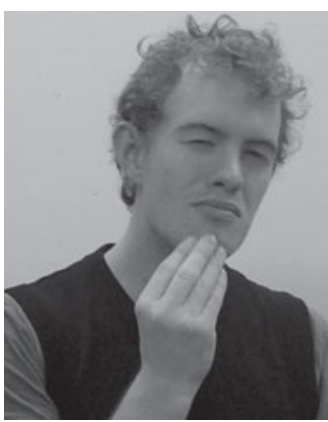

Brown-ish

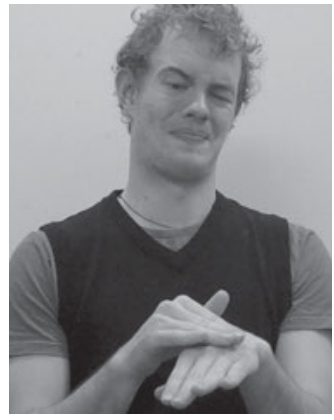

Blue - intense/vivid

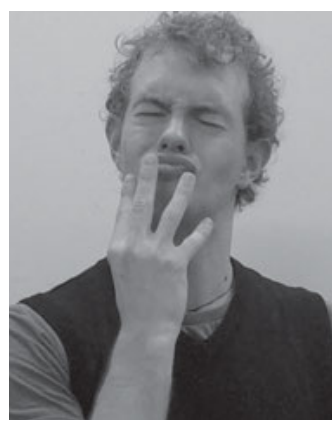

Brown - dark

Figure 23. Non-manual modification of colours 


\subsection{The semantic origin of colour signs}

The generic NZSL colour sign is not transparently motivated. Folk etymology would suggest that contemporary colour signs borrowed from Auslan (with roots in BSL) are semantically motivated by reference to coloured objects, as follows: Blue - originally veins on the underside of wrist, later shifted to back of hand (Woll 1987); Black - associated with dark skin colour, and perhaps cleaning dirt/coal off the face; White - shirt collar (perhaps originally neck garments); Yellow - gold earring or blonde hair; Red - lips; Brown - beard; Orange - the sign also names the fruit, which is squeezed; Green - may derive from BSL signs 'park/field' and 'grass' which are formationally similar; Grey - possibly the colour of a man's cheek with 'five o'clock shadow'. There is little empirical evidence available to verify these speculations about etymology, but they seem plausible.

NZSL has no colour signs that are initialised or based on orthography, except possibly one archaic variant of green, which appears to depict the hook of lower case ' $\mathrm{g}$ ' (see figure 24).

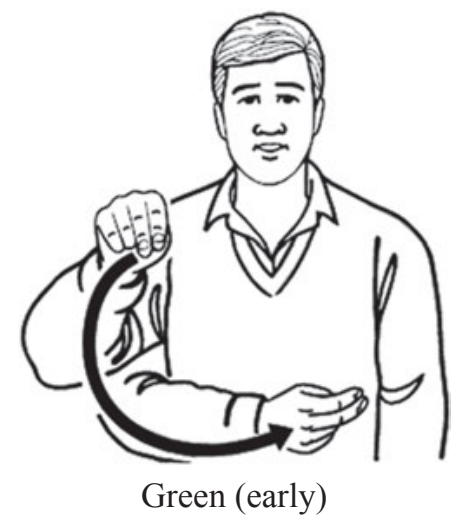

Figure 24. Green (early form)

NZSL signers do not use pointing to real objects as a regular strategy for colour reference. However, the locatability of signs allows for the association of colour terms with a specific location or object. NZSL signs for hair colours, blonde and grey, contain a deictic location feature, being produced at the head. Blonde has two variants, both located 'on' the hair: an earlier form (fingertips of a bent-five hand stroke the hair downwards, twice) and a modern (Auslan) variant that is apparently a compound of YELLOW'HAIR. 'Greyhair' is made by spreading the open fingers back over the hair in a movement starting from the temple, suggesting the spread of grey hair from the temples, 
(see figure 25a). The concept of 'grey (overcast) sky' (see figure 25b) is expressed by the open hands depicting sky as if it were a coloured surface; the handshape corresponds closely with the general sign for colour, also used in 'weather' (figure 25c), located iconically towards the sky.

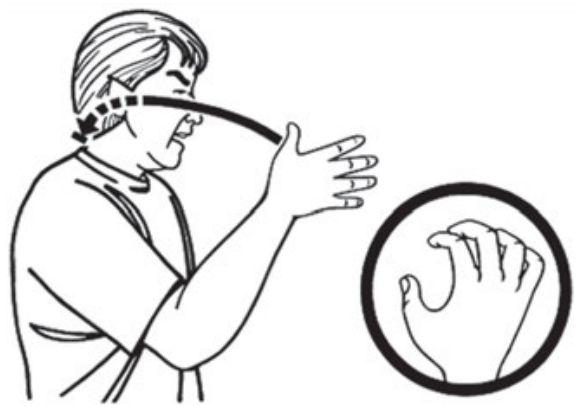

(a) grey haired

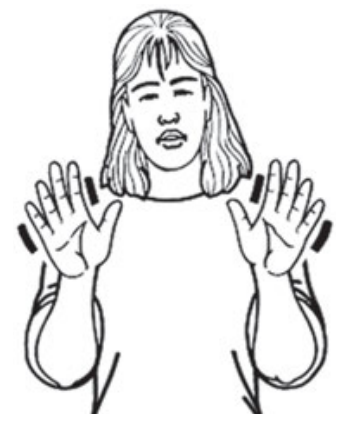

(b) grey, cloudy

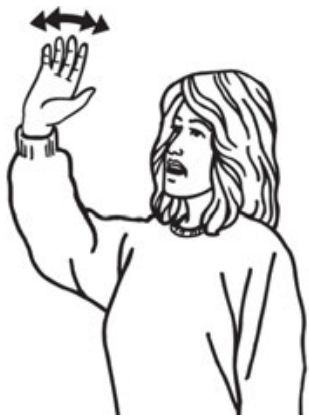

(c) weather

Figure 25. Object colour reference

The deictic element of location in hair colour signs is also present in signs for skin shades, blush and pale, which are produced in contact with the cheek where these shades are normally observed.

\section{Kinship}

Relationships between kin are expressed through conventional lexicon in NZSL, rather than in diagrammatic signing space. A search in NZSL Online under the semantic domain 'family' returns 56 entries; the relatively large number of signs tagged to this domain in the dictionary is expanded partly by the inclusion of family-related concepts such as 'adopt', 'foster', 'divorce', 
'twins', 'wedding', 'family history'. The number is also increased by the existence of variants for many kin terms. 'Dad', for example, has three phonological variants, all modifications of a borrowed sign formed with a one-handed (ASL) 'D' handshape which originates in American Signed English. 'Nephew' also shows three variants, of which two are phonological variants of a nonnative sign with initialisation from a fingerspelling system.

Like number and colour signs, diachronic variation in kinship vocabulary is differentiated by pre- and post-Australasian Signed English eras in education. The earlier paradigm utilised a generic sign indicating a relationship, with reference specified by mouthing a kinship word, (see 'Relation' in figure 26). This sign also means 'same', 'friend', 'girlfriend', 'boyfriend' (in pre-ASE lexicon), 'relative', and serves as the dual person pronoun. These meanings are specified by mouthing and context. Older signers use this generic relationship sign to refer to all family members (including husband/wife), except mother and father, which had distinct signs in the early lexicon. In this paradigm 'parents' is expressed by a compound of MOTHER ${ }^{\wedge}$ FATHER. The early paradigm sign for 'family' is also a polysemous form that refers more generally to a group. The early paradigm of kinship signs is shown in figure 26 .

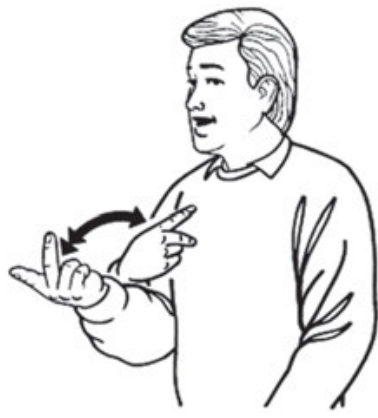

Relation

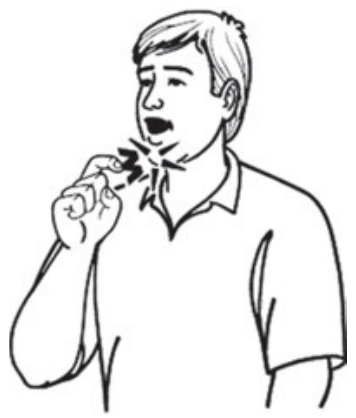

Father

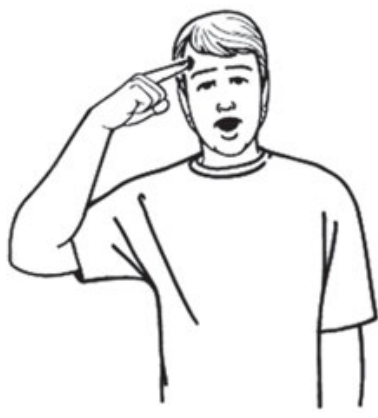

Mother

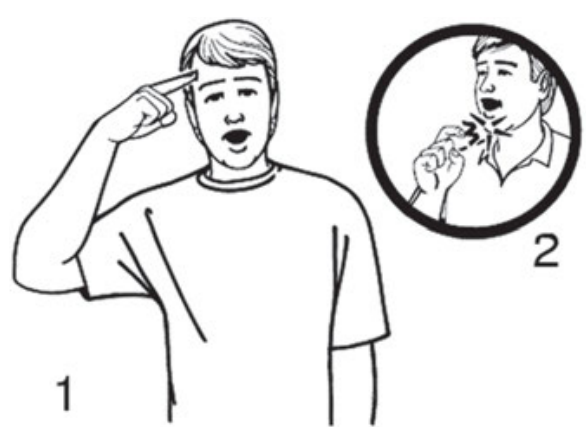

Parents

Figure 26. Early kinship signs 
A new set of kinship signs entered NZSL via ASE from 1979, as illustrated in Table 1. These are now established in NZSL and almost universally used by signers below the age of about 55 years (and many above). Analysis of variation in the use of kinship signs across three age cohorts of NZSL signers, shows that use of the generic 'relative' sign, however, is not entirely confined to older signers, but is rather in a state of progressive semantic change. Whereas older signers, on the whole, favour this form to express nearly all kin (and friend) relationships, middle-aged signers still commonly use it for niece, nephew, aunt and uncle, while younger signers use it regularly only to mean 'relatives' and as one variant of son. The semantic scope of the sign is thus progressively narrowing, as more formally and semantically distinct alternatives have been added to the language (McKee and McKee 2011).

The current paradigm of family terms is conceptually consistent with that of English speaking cultures (see Table 1); there are no lexical distinctions between older/younger or paternal/maternal relatives. Gendered signs correspond with gender-specified terms in English: grandmother/grandfather, mother/father, daughter/son, sister/brother, aunt/uncle, niece/nephew. However, the manual sign for husband/wife is not gender differentiated except by mouthing of the relevant word (not always correctly selected; indeed, on a visit to the Deaf club on the day of this writing, the married female author was asked in NZSL (with mouthing), "Is your 'wife' here tonight?").

Fingerspelling features prominently in the formation of modern (ASE/ Auslan-origin) signs for family members. Several core terms are single manual letter signs; e.g., M for mother, F for father, D for daughter, S for son, G-F for grandfather, G-M for grandmother. Except S for son, these are also found in BSL. Others (family, aunt, uncle, cousin, niece, nephew, in-law) are formed with initialised handshapes in signs that were introduced with ASE. Of the core terms in this set, only brother, sister and spouse do not include a fingerspelled element. (See illustrations in Table 1.) The presence of fingerspelling handshapes in this modern lexicon sets it clearly apart from the earlier system of kinship reference in NZSL, in which mouthing (contextualised by the 'relationship' sign) was the mode of borrowing English terms. Prior to the introduction of ASE into schools in 1979, fingerspelling was not generally used in the deaf community except by individual immigrants from the UK or Australia, and it had no presence in the early lexicon.

A collective term for parents is a compound MOTHER $\wedge$ FATHER; the concept of siblings is expressed by juxtaposed signs BROTHER, SISTER but the combination is neither highly frequent nor has phonological reduction typical of a lexicalised compound. Half, step, and in-law relationships are identified with multi-sign phrases that correspond in structure with English 
terms - e.g., HALF BROTHER, STEP FATHER, MOTHER IN-LAW, (see Table 2). Complex constructions expressing relationships such as 'father's sister' or 'wife's cousin' can be expressed as juxtaposed signs (FATHER SISTER), or linked by the possessive pronoun (FATHER HIS SISTER); both strategies are common.

In discourse, signs for family members may be indexed to loci in the signing space for anaphoric reference, but morphological strategies of reduplication or spatial modification to express meanings of pluralisation, or generational or age-related hierarchy are not encoded in the formation of signs.

Sibling birth order is conveyed by modifiers 'older' (literally TALL) and 'younger' (literally SHORT), or by indexing ordinal list-buoys on the extended fingers of the non-dominant hand.

Table 1. Core family signs: modern paradigm

\begin{tabular}{cl}
\hline \multicolumn{1}{c}{ Category } & \multicolumn{1}{c}{ Gloss } \\
\hline Non-gendered parent(s) & $\begin{array}{l}\text { Compound of } \\
\text { gendered terms: } \\
\text { MOTHER FATHER }\end{array}$ \\
Gendered parent & FATHER \\
Nendered parent & CHILD \\
Gendered-offspring & DAUGHTER
\end{tabular}


Gendered-offspring

Gendered sibling

Gendered sibling

Non-gendered spouse
SON
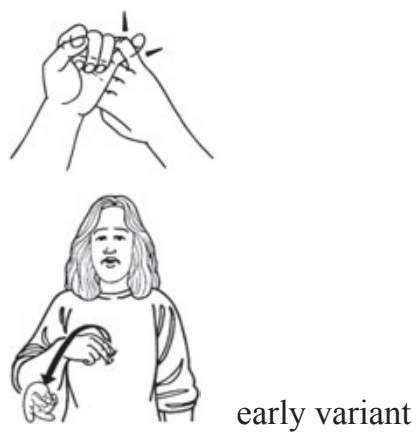

BROTHER

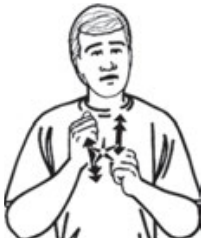

SISTER
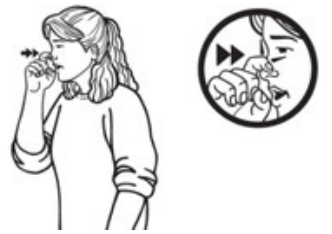

SPOUSE

(gender specified by mouthing)

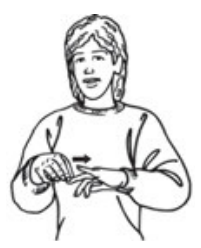

Table 2. Non-core family terms: modern paradigm

\begin{tabular}{ccc}
\hline Term & Sign gloss & Picture \\
\hline Gendered grandparent & GRANDMOTHER
\end{tabular}

Gendered grandparent GRANDFATHER

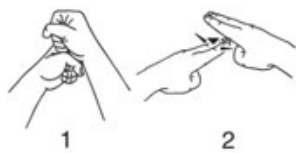


Non-gendered

grandchild

Gendered grandchild GRANDSON

Gendered grandchild

Non-gendered aunt/ uncle (ego's parents' siblings)

Gendered ego's parents' siblings

Gendered ego's parents' siblings

RELATIVE

AUNT

UNCLE
GRANDCHILD
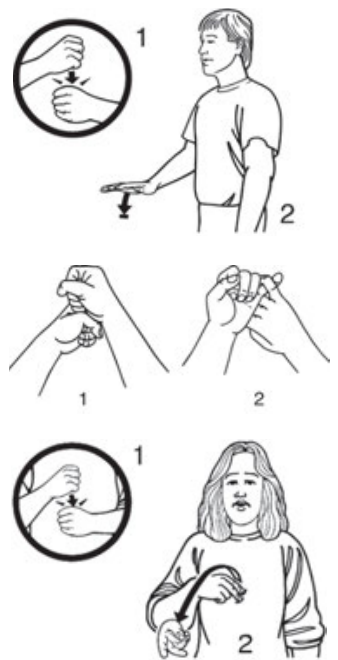

GRANDDAUGHTER

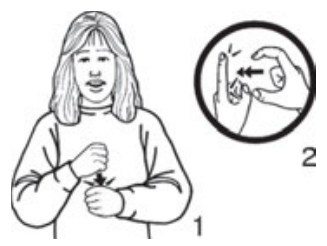

used by older signers

for aunt, uncle,

specified by mouthing
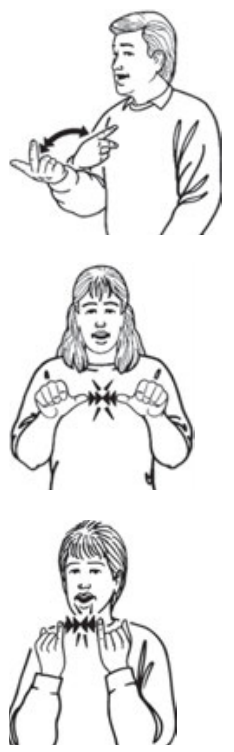
Non-gendered cousin (same generation)

Non-gendered niece/ nephew (children of ego's sibling)

Gendered children of ego's sibling (female)
COUSIN

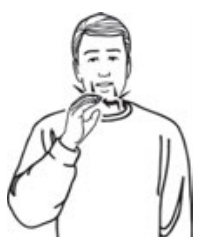

RELATIVE used by older signers for aunt, uncle, specified by mouthing

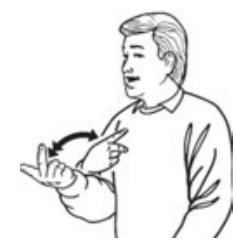

NIECE

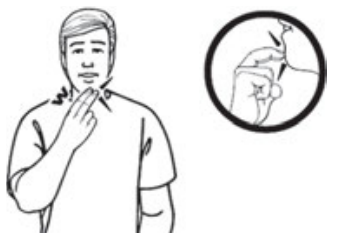

Gendered children of NEPHEW ego's sibling (male)

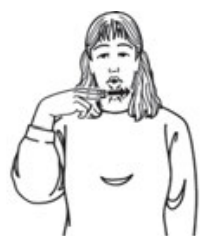

Non-gendered ENGAGED, engagement relation FIANCE(E)

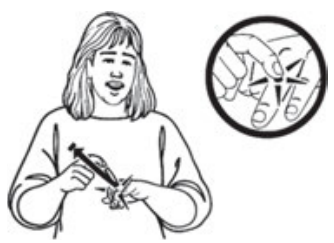




\section{Conclusion}

Major cultural and linguistic change within signed languages is often precipitated by macro and micro developments in deaf education. In relation to signed languages, Stokoe (1987:9) notes that "the fact of major cultural change may be approximately dated by the extent of colour vocabulary". The hybrid character of the three semantic domains surveyed in this chapter, number, colour and kinship reflects an abrupt change in the linguistic resources available to deaf people in New Zealand, dating from the 1979 switch from oralism to the use of Australasian Signed English in education. This intervention differentiated the core vocabularies of younger and older generations of signers, and has resulted in progressive decline in the use of signs from an earlier paradigm of NZSL.

Both early and contemporary systems of colour and kinship terms contain non-native elements: earlier, through the mouthing of English words to specify reference with generic signs, and in the modern paradigm, through Auslan signs imported via Australasian Signed English. In the domain of kinship, new vocabulary introduced alphabetic handshapes in initialised signs, previously absent in NZSL phonology. In the case of numbers, systems of morphological inflection have been extended, and appear to be still developing among signers of middle and younger generations. Thus, the adoption of Auslan vocabulary for number, colour and kinship concepts has altered the size and the formational parameters of the NZSL lexicon. The intended purpose of introducing ASE in New Zealand education was to increase parallelism between a signed lexicon and spoken English, which was achieved to some extent; a less intentional, but significant outcome in cultural and linguistic terms, was setting NZSL on a path towards increasing convergence with its historical relatives, Auslan and BSL. 


\section{Acknowledgements}

Thanks to Mark Berry and Sara Pivac Alexander, native NZSL consultants. Mark kindly modelled the photographic illustrations. All line drawings are by Shaun Fahey, reproduced with permission from the Online Dictionary of NZSL (http://nzsl.vuw.ac.nz/). Thanks to Adam Schembri for helpful comments on relationships between certain NZSL, Auslan and BSL signs.

\section{Notes}

1. The term 'Australasian' refers jointly to Australia and New Zealand.

2. The earlier Auslan usage was pointed out to me as a possible source by Adam Schembri. Pounds were replaced with decimal currency (dollars) in Australia in 1966 and 1967 in New Zealand. While this 'pound' incorporating form reportedly still occurs in some older Auslan users, there is no documented evidence of it in a similar generation of NZSL users, although it is possible that it entered NZSL via contact with older Australians. Borrowing from current BSL seems more likely since this form is seen more among young generation NZSL signers, some of whom have worked and lived in the UK.

3. For example, a hearing daughter of deaf parents of the generation who used this colour system reported that one of her parents consistently mouthed something like 'ruthuh' with the generic colour sign, to mean 'red'. Her other parent would always point to the nose for 'red', which she only discovered as an adult was a 'family sign', not a conventional NZSL sign. 


\section{References}

Collins-Ahlgren, Marianne. 1989. Aspects of New Zealand Sign Language. Unpublished doctoral thesis, Victoria University of Wellington, New Zealand.

Fitzgerald, Sarah. 1999. Open Minds, Open Hearts: Stories Of The Australian Catholic Deaf Community. Lidcombe, NSW: CCOD.

Forman, Wayne. 2003. The ABCs of New Zealand Sign Language: Aerial Spelling. Journal of Deaf Studies and Deaf Education, 8(1), 92-96.

Fox, Margalit. 2007. Talking hands. What sign language reveals about the mind. New York: Simon and Schuster.

Hollman, Liivi and Urmas Sutrop . 2011. Basic Colour Terms in Estonian Sign Language. Sign Language Studies 11(2), 130-157.

Hoyer, Karin. 2007. Albanian Sign Language: Language Contact, International Sign, and Gesture. In Sign Languages in Contact. David Quinto-Pozos (ed.), 195-234. Washington, D.C.: Gallaudet University Press.

Jeanes, Raymond C., Brian E. Reynolds and Bernadette C. Coleman (eds.). 1982. Dictionary of Australasian Signs for Communication with the Deaf. Second Edition. Brunswick, Victoria: Globe Press.

Johnston, Trevor. 2003. BSL, Auslan and NZSL: Three signed languages or one? In Cross-Linguistic Perspectives in Sign Language Research, Selected papers from TISLR 2000.Anne Baker, Beppie van den Bogaerde and Onno Crasborn (eds.), 47-69. Hamburg: Signum Verlag.

Johnston, Trevor and Adam Schembri. 2007. Australian Sign Language. An Introduction to Sign Linguistics. Cambridge: Cambridge University Press.

Kennedy, Graeme, Richard Arnold, Patricia Dugdale, Shaun Fahey, and David Moskovitz. 1997. A Dictionary of New Zealand Sign Language. Auckland: Auckland University Press with Bridget Williams Books.

Levitt, Dan. 1986. Introduction to New Zealand Sign Language. Auckland: Deaf Association and National Foundation for the Deaf.

McKee, David and Graeme Kennedy. 2000. A lexical comparison of signs from American, Australian, and New Zealand Sign Languages. In The Signs of Language Revisited, Karen Emmorey and Harlan Lane (eds.), 49-76 Mahwah, NJ: Lawrence Erlbaum Associates.

McKee, Rachel and David McKee. 2011. Old signs, new signs, whose signs? Sociolinguistic variation in the New Zealand Sign Language lexicon. Sign Language Studies 11(4), 485-527.

McKee, David, Rachel McKee and George Major. 2011. Numeral variation in New Zealand Sign Language. Sign Language Studies 11 (5), 72-97 
Nyst, Victoria . 2007 Simultaneous constructions in Adamorobe Sign Language. In Simultaneity in signed languages: Form and function. Myriam Vermeerbergen, Lorraine Leeson and Onno Alex Crasborn (eds.),127-146. Amsterdam and Philadelphia: John Benjamins.

Schembri, Adam, Kearsey Cormier, Trevor Johnston, David McKee, Rachel McKee and Bencie Woll. 2010. British, Australian, and New Zealand sign languages: Origins, transmission, variation and change. In Sign Languages: A Cambridge Language Survey. Diane Brentari (ed.), 476-498. Cambridge University Press.

Stokoe, William C. 1987. Lexical indicators of cultural change. In Sign and School. Jim G. Kyle (ed.),7-11. Clevedon UK: Multilingual Matters.

Washabaugh, William. 1986. Five Fingers for Survival. Ann Arbor, Mich.: Karoma.

Woll, Bencie. 1987. Historical and Comparative Aspects of BSL. In Sign and School. Jim G. Kyle (ed.), 12-34. Clevedon UK: Multilingual Matters 